J. Lake Sci. (湖泊科学), 2011, 23(5): 783-788

http: //www. jlakes.org. E-mail: jlakes@niglas.ac.cn

(C) 2011 by Journal of Lake Sciences

\title{
淀山湖风浪场的数值模拟”
}

\author{
张洪生, 幸俊波, 文武健 \\ (上海交通大学海洋工程国家重点实验室,上海 200240)
}

\begin{abstract}
摘 要: 对淀山湖的风场和风浪场进行了观测, 根据所观测的风场利用 SWAN 模型计算了湖区的风浪场. 通过将计算的 波高和观测值进行比较, 说明计算结果的变化趋势和观测结果的变化趋势相吻合; 观测结果的波动幅度较大, 而计算结 果比较平滑. 总体说来,基于 SWAN 模型所计算的波高可信、可靠. 在此基础上,利用 SWAN 模型较为系统地计算了不同 水位和不同风速的风场组合作用下淀山湖水域的风浪场,为今后淀山湖的防汛和环境保护提供了初步的参考依据.
\end{abstract}

关键词: 淀山湖; 风浪场; SWAN 模型; 数值模拟

\section{Numerical simulation of the wind wave fields in Lake Dianshan}

\author{
ZHANG Hongsheng, GU Junbo \& WEN Wujian \\ (State Laboratory of Ocean Engineering, Shanghai Jiaotong University, Shanghai 200240, P. R. China)
}

\begin{abstract}
On the basis of observation for wind fields and wind wave fields in Lake Dianshan, the wind wave fields were numerically simulated with the SWAN model. Comparisons of wave height between modeling and measurement indicated that the variation tendencies of the modeled wind wave fields were in agreement with those of the measured data. However, the fluctuation amplitudes of measured data are greater than those of modeled results. On the whole, the modeled wind wave fields are reasonable and reliable. Therefore, the wind wave fields in Lake Dianshan have been systematically simulated with different water levels and different wind fields. The modeled results can be taken as the reference basis for the flood prevention and environmental conservation of Lake Dianshan.
\end{abstract}

Keywords: Lake Dianshan; wind wave field; SWAN model; numerical simulation

淀山湖属太湖流域, 为平原浅水湖泊, 呈东北-西南走向, 南宽北窄, 形似葫芦. 其长度为 $14.5 \mathrm{~km}$, 最大 宽度为 $8.1 \mathrm{~km}$, 平均宽度为 $4.3 \mathrm{~km}$, 岸线长为 $62.3 \mathrm{~km}$, 多年平均水位为 $2.63 \mathrm{~m}$ (城建吴淞高程, 下同), 其面 积为 $63 \mathrm{~km}^{2}$, 属于平原浅水湖泊, 具有风生浪起的特征. 夏秋季节在东南季风的影响下, 降水量大, 导致水位 暴涨, 对其风浪场进行研究对于防台和防汛都具有十分重要的意义. 而且, 对于水质的评价也是重要的, 因 为风浪对水流的作用通过辐射应力的形式表现出来,从而影响和改变水流的结构.

研究湖泊的风浪特性, 可以采用三种方法. 一种是现场观测的方法, 例如有研究对太湖的风浪进行了观 测 $^{[1-3]}$. 通过该方法获得的数据资料可靠性大. 但通过现场观测的办法不可能提供整个水域的风浪场, 而且 在极端天气状况下往往较难观测到风浪的波要素 (极端天气有可能造成观测设备的损坏). 因此,不可能对 其在各种风向、风力条件下的全水域进行观测. 第二种方法是利用经验公式计算湖区内工程位置的波要素. 《堤防工程设计规范》(GB50286-98 $)^{[4]}$ 规定了计算内陆湖泊风浪要素的方法. 该计算方法需假定湖区的水 深和风场变化比较缓慢. 但事实上湖区的水深并不一定缓慢变化,尤其是岸堤附近水深变化较大; 风场随时 空的变化也并不一定缓慢,尤其是遇到台风过程时, 不同位置的风向可能有很大的不同. 应用规范规定的方 法计算内陆湖泊的风浪究竟存在多大误差, 还需要进行进一步的定量研究. 而且, 虽然该方法在计算单个工 程位置的波要素时较为简单,但采用该方法很难较快地计算出整个湖区在不同时刻的波要素. 根据风浪生

* 国家自然科学基金项目 (40676053,51079082)、上海市科学技术委员会项目 (08DZ1203005) 和上海交通大学海洋 工程国家重点实验室开放基金项目 (GKZD010012, GP010818, GKZD010024) 联合资助. 张洪生, 男, 1967 年生, 博 士,副教授;E-mail:hhszhang@sjtu. edu.cn. 
成数学模型对风浪生成、成长和衰减过程进行数值模拟, 是风浪预报计算的一种理论性较严密、考虑天然影 响因素较多的方法. 风浪生成、成长、传播及衰减过程涉及大气与水面相互作用、波浪动力学特性等复杂问 题. 天然风浪具有随机性、频散性和非线性等特性, 是一种随时间、空间变化的随机过程 ${ }^{[5]} .1940 \mathrm{~s}$ 蒙克等人 提出特征波波能平衡方程, 开创了风浪特征波要素 (如有效波高与有效波周期或平均波高与平均波周期) 的 数值模拟新途径. $1960 \mathrm{~s}$ 前后, 海斯曼 ${ }^{[6-8]}$ 提出二维谱平衡方程, 他们结合风浪生成共振理论和切变流不稳定 理论对具有二维波能谱的随机风浪进行数值模拟, 提供了预报风浪谱及其谱特征的新方法. 由于谱分量平 衡方程中所包含的波波非线性相互作用源项涉及到 6 维的 Bottzmann 积分, 计算极其困难, 因此, 各国学者 先后提出不同的处理方法. 至今, 已发展到了第三代模式. 第一代模式不考虑波波非线性相互作用,第二代 模式是以参数化简化方法考虑波波非线性相互作用, 第三代模式是采用与离散化自由度相同参数直接计算 非线性源项 ${ }^{[9]}$. SWAN (Simulating Waves Nearshore) 模型 ${ }^{[10]}$ 是荷兰 Delft 理工大学 (Delft University of Technology) 基于第三代模式建立的风浪数值模型, 该模型综合考虑了波浪传播过程中水底地形的折射、波浪破碎、 底摩擦、波波相互作用和白帽浪等多种因素的影响.

SWAN 模型是国际上比较通用的近岸风浪模型. 但该模型还较少应用于内陆湖泊风浪的数值模拟 ${ }^{[1]}$. 相对于近岸水域,一般内陆湖泊的岸线更为曲折复杂, 水深变化也更为剧烈. 而 SWAN 模型存在着不能有效 模拟近边界处的波浪场的缺点. 这就需要我们将该模型应用于内陆湖泊风浪的数值模拟时要特别注意边界 的处理. 本文首先将 SWAN 模型应用到淀山湖水域风浪的数值模拟, 并将数值模拟结果和现场观测值进行 比较, 然后对淀山湖水域的风浪场进行较为系统的数值模拟.

\section{SWAN 模型的原理简介}

SWAN 模型 ${ }^{[10]}$ 是根据风、底部和水流的条件对近海地区、湖泊和河口的波浪进行数值模拟的第三代波 浪模型. 该模型的控制方程是基于带有源、汇项的波作用平衡方程,其在直角坐标系下的表达形式为:

$$
\frac{\partial N}{\partial t}+\frac{\partial}{\partial x} C_{x} N+\frac{\partial}{\partial_{y}} C_{y} N+\frac{\partial}{\partial \sigma} C_{\sigma} N+\frac{\partial}{\partial \theta} C_{\theta} N=\frac{S}{\sigma}
$$

式中, $N(\sigma, \theta)=E(\sigma, \theta) / \sigma, \sigma$ 为相对频率, $\theta$ 为波向, $E(\sigma, \theta)$ 为能谱密度, $N(\sigma, \theta)$ 为波作用量; $C_{x} 、 C_{y} 、 C_{\sigma}$ 和 $C_{\theta}$ 分别为波作用量在 $x$ 方向、 $y$ 方向、频率空间和波向空间中的传播速度; $S$ 代表物理过程所产生的源 汇项.

模型主要考虑的物理过程是: 地形影响所产生的折射和浅水变形、非线性的波波相互作用(包括浅水的 三个一组和深水的四个一组相互作用)、障碍物透射,并初步考虑绕射作用. 方程的源、汇项主要包括: 风能 摄人、白帽浪的能量耗散、水深所引起的波浪破碎、底摩擦损耗等. 模型能输出波高、波周期和波长等多种物 理量, 能够根据需要灵活选用所需要考虑的物理过程以及某一物理过程的不同参数化形式.

研制 SWAN 模型的初衷是模拟水深相对较浅的河口海岸水域内风浪的生成、传播和变形,该模型自从 问世以来得到了迅速发展, 到目前为止, 它仍处于不断发展过程中, 并在近岸水域得到了较好的应用 ${ }^{[12-16]}$. 同时,也有将其应用于模拟内陆湖泊风浪的算例 ${ }^{[11]}$.

\section{SWAN 模型有关参数的选取}

SWAN 模型中包含有若干物理过程的处理, 相应地含有不少计算参数. 但模型手册强烈建议使用模型 系数的缺省值. 我们在数值计算淀山湖的风浪场时, 也曾经对若干参数进行了调试, 例如调试了底摩擦系 数, 但发现对计算结果的影响很小, 当然, 这有可能与我们计算的情况有关. 鉴于该模型是一个使用广泛的 模型, 在数值计算淀山湖的风浪场时, 对于物理过程的处理我们使用了 SWAN 模型建议的相关参数值. 其它 相关参数主要有: $\theta$ 空间的网格数采用 36 , 即谱方向的分辨率为 $10^{\circ}$; 最小频率为 $0.08 \mathrm{~Hz}$ 、最高频率为 $1.0 \mathrm{~Hz}$; JONSWAP 底摩擦公式的系数取为 $0.067 \mathrm{~m}^{2} / \mathrm{s}^{3}$. 当波高的最大值与水深的比值为 0.73 时允许波浪 破碎. 数值求解格式采用一阶的空间和时间向后差分格式 (BSBT), 每个时间步的最大迭代次数选为 3 次. 经 我们的测试说明, 迭代次数选为 3 次是恰当的 ${ }^{[3]}$. 如迭代次数少于 3 次, 有可能产生计算结果的不稳定; 如 迭代次数大于 3 次,并不会提高和改善计算的精度. 
经过模型试验, 将包含整个湖区的计算域沿 东西方向剖分为 211 个网格点, 沿南北方向剖分 为 232 个网格点, 网格步长均取为 $100.0 \mathrm{~m}$. 图 1 为湖底地形高程图, 将读人点的水深插值到计算 网格点上,公式为:

$$
d_{i, j}=\sum_{m=1}^{4} \omega_{m} d_{m}, \omega_{m}=\frac{S_{m}^{-1}}{\sum_{m=1}^{4} S_{m}^{-1}}
$$

式中, $S_{m}$ 为坐标点 $(i, j)$ 周围距离最近的四个节点 之一的第 $m$ 个节点到坐标点的距离, $\omega_{m}$ 为其权 重, $d_{m}$ 为第 $m$ 个节点的水深, $d_{i, j}$ 为坐标点 $(i, j)$ 处 的水深.

\section{3 计算结果的验证}

我们在湖区三个测点位置进行了现场测量, 其中 $1^{\#}$ 站位的坐标为 $\left(31^{\circ} 5^{\prime} 11.5^{\prime \prime} \mathrm{N}, 120^{\circ} 55^{\prime} 29.9^{\prime \prime} \mathrm{E}\right), 2^{\#}$ 站位的坐标为 $\left(31^{\circ} 6^{\prime} 6.3^{\prime \prime} \mathrm{N}, 120^{\circ} 56^{\prime} 50.1^{\prime \prime} \mathrm{E}\right), 3^{\#}$ 站 位的坐标为 $\left(31^{\circ} 7^{\prime} 22.1^{\prime \prime} \mathrm{N}, 120^{\circ} 58^{\prime} 13.2^{\prime \prime} \mathrm{E}\right)$. 观测 时间为 2009 年 7 月 18 日和 21 日两个白天. 风浪

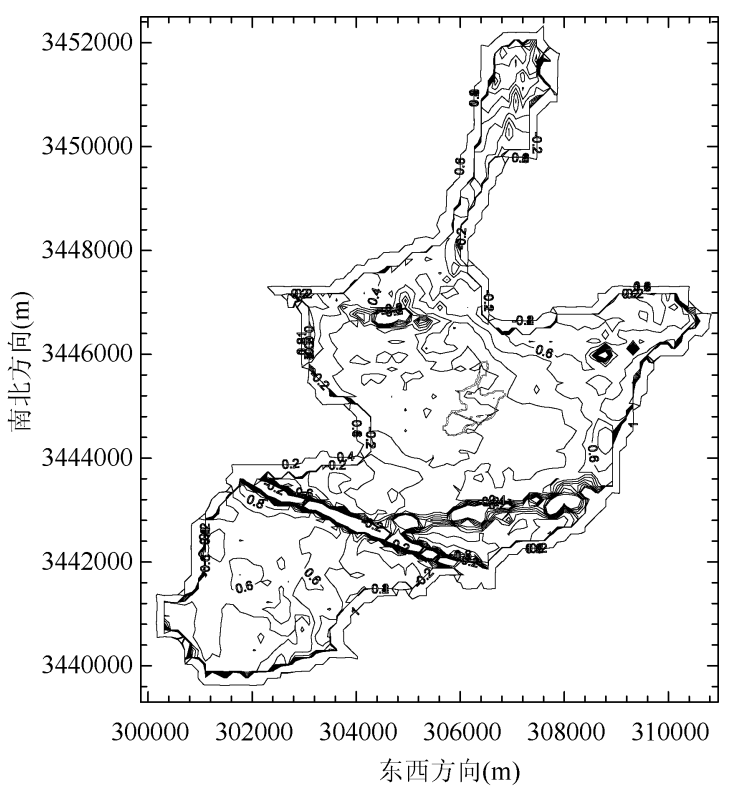

图 1 湖底地形高程图

Fig. 1 The elevation chart at Lake Dianshan bed 观测仪器采用美国海鸟 (Seabird) 公司生产的浪 潮仪 SBE-26. 该仪器适用的测量水深范围为 0-21.0 $\mathrm{m}$ 、水温范围为 -5 至 $45^{\circ} \mathrm{C}$; 采样时间间隔为 $0.25 \mathrm{~s}$ 时, 波高的分辨率为 $0.4 \mathrm{~mm}$. 我们每 $20 \mathrm{~min}$ 观测一次, 每次观测的持续时间为 $256 \mathrm{~s}$ 或 $512 \mathrm{~s}$, 采样时间间隔为 $0.25 \mathrm{~s}$. 我们将浪潮仪悬吊在三角支架上,并沉人水下.

在同一个时刻, 湖中三个站位的风场并不相同, 它们随时间的变化也并不同步 (图 2 和图 3 ). 在我们进 行测量的时间段内风力很弱, 可以想象, 在遇到台风过程或强对流天气时, 风场的时空变化会更为剧烈, 此 时三个站位风场的差别会更大.
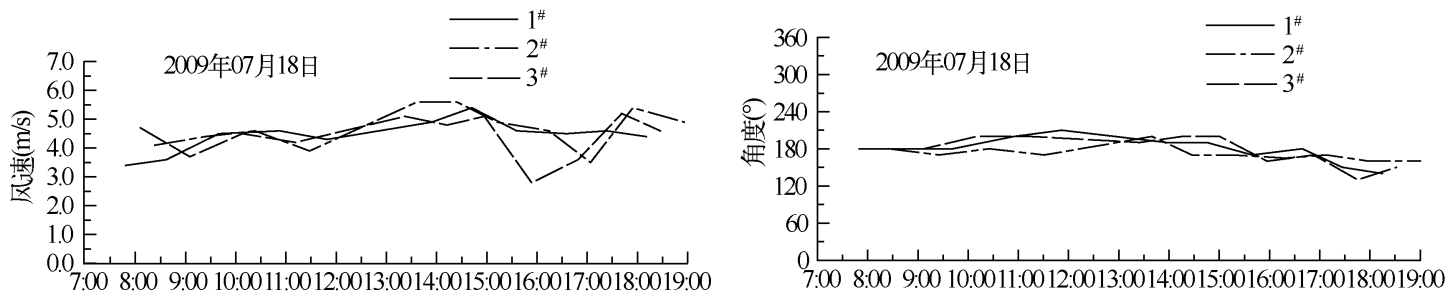

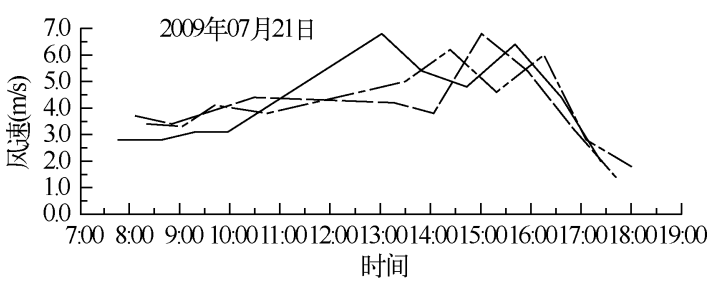

图 2 观测的三个站位的风速

Fig. 2 The measured wind speed at three locations

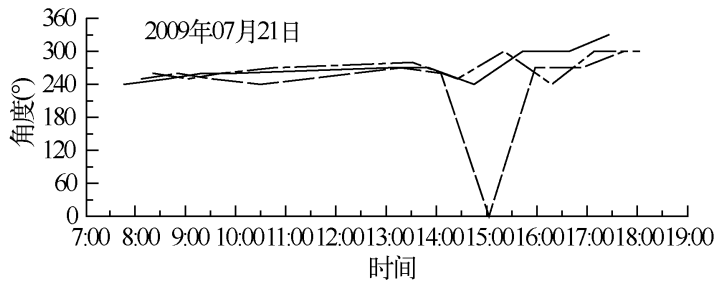

图 3 观测的三个站位的风向

Fig. 3 The measured wind direction at three locations

我们曾经在计算范围为 $40 \times 40 \mathrm{~km}$ 的水域, 就风速为 $10 \mathrm{~m} / \mathrm{s}$ 的情况, 探讨了利用非恒稳模式进行计算 时, 波高随时间的变化过程. 结果发现, 在 $100 \mathrm{~min}$ 后, 所计算的波高和稳态模式的计算结果接近 ${ }^{[3]}$. 李一平 
等在基于 SWAN 模型的非稳态模式计算太湖水域的波浪时,通过假定不同的风速也探讨了波高随时间变化 的特点, 发现在风速为 $5 \mathrm{~m} / \mathrm{s}$ 的时候, 风浪发展到稳定需时间 $120-150 \mathrm{~min}^{[11]}$. 鉴于淀山湖的水域面积远远 小于太湖 (太湖的水域面积为 $2338.11 \mathrm{~km}^{2}$ ), 我们在模型运行 $2 \mathrm{~h}$ 后, 输出计算结果应该是完全可行的.

鉴于 SWAN 模型在开始的一段时间内的计算结果不可靠, 我们从 7:00 开始进行计算, 从 7:00 至 9:00 之间的风场采用 9:00 的观测数据. 在 7 月 18 日, $1^{\#}$ 站位、站位和 $3^{\#}$ 站位的绝对误差分别为 $0.04 、 0.05$ 和 $0.08 \mathrm{~m}$,三个观测站的平均绝对误差为 $0.06 \mathrm{~m} ; 1^{\#}$ 站位、 $2^{\#}$ 站位和 $3^{\#}$ 站位的相对误差分别为 $19.99 \%$ 、 $24.57 \%$ 和 $27.48 \%$,三个观测站的平均相对误差为 $24.01 \%$. 在 7 月 21 日, $1^{\#}$ 站位、 $2^{\#}$ 站位和 $3^{\#}$ 站位的绝对 误差分别为 $0.02 、 0.05$ 和 $0.04 \mathrm{~m}$,三个观测站的平均绝对误差为 $0.04 \mathrm{~m} ; 1^{1}$ 站位、 $2^{\#}$ 站位和 $3^{\#}$ 站位的相对误 差分别为 $17.91 \% 、 28.48 \%$ 和 $22.02 \%$,三个观测站的平均相对误差为 $22.80 \%$. 在总计两天的时间内,波高 的计算值和观测值的平均绝对误差为 $0.05 \mathrm{~m}$, 平均相对误差为 $23.41 \%$. 由于在两天的时间内, 观测的最大 风力均不超过 4 级, 由此生成的浪高也很小, 最大观测值为 $0.35 \mathrm{~m}$. 这样就会导致相对误差比较大. 由图 4 和图 5 可见,计算结果的变化趋势和观测结果的变化趋势相吻合. 但二者也存在差别: 实测值的波动较大, 而计算值的波动较小. 我们认为产生差别的原因应当是: 风浪本身具有随机性和瞬时性, 而且波高本身不 大, 如有外来扰动, 很容易引起测量值的改变; 而利用 SWAN 模型计算风浪场时, 所采用的风场是 3 个观测 站点的平均值, 而且所得到的波高是数值积分的计算结果, 因此其波动幅度比较小. 总体说来, 利用 SWAN 模型所计算的有效波高定性正确、定量可靠.
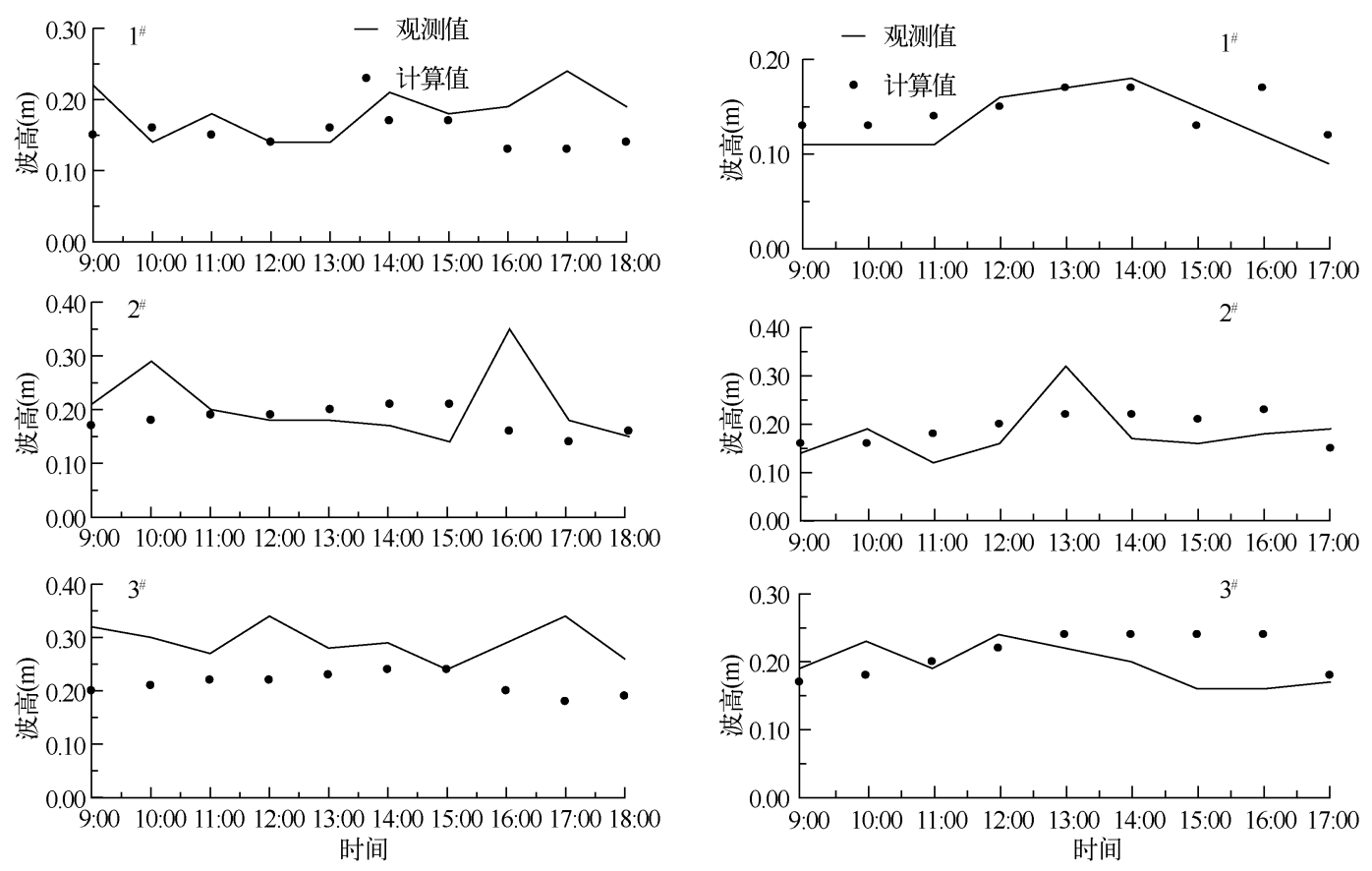

图 42009 年 07 月 18 日的波高计算结果 与实测结果的比较图

Fig. 4 The comparison of calculated wave height with the measured data on July 18,2009

图 52009 年 07 月 21 日的波高计算结果 与实测结果的比较图

Fig. 5 The comparison of calculated wave height with the measured data on July 21,2009

\section{4 不同条件下淀山湖风浪场的数值模拟}

\section{1 夏季不同方向风场作用下风浪场的数值模拟}

鉴于夏季 (6月-8 月) 是淀山湖的汛期,受东南季风的影响, 我们对夏季不同风向的风场作用下淀山湖 的风浪场进行模拟研究. 因为淀山湖属于太湖流域, 所以参考太湖的风场统计资料 ${ }^{[17]}$ 确定淀山湖的风场: 在 
夏季以 ESE、SSE 和 SSW 风向较多. 采用 $2 、 4 、 6$ 和 7 四个风级进行模拟. 根据文献 ${ }^{[18]}$ 的风力分级标准, 2 级 风的风速范围为 $1.6-3.3 \mathrm{~m} / \mathrm{s} ; 4$ 级风的风速范围为 $5.5-7.9 \mathrm{~m} / \mathrm{s}$; 6 级风的风速范围为 $10.8-13.8 \mathrm{~m} / \mathrm{s}$; 级风的风速范围为 $13.9-17.1 \mathrm{~m} / \mathrm{s}$; 它们的中位数分别为 $2.5 、 6.7 、 12.3$ 和 $15.5 \mathrm{~m} / \mathrm{s}$. 在计算各级风力时, 采 用中位数进行计算. 水位取其夏季的多年平均值 $2.85 \mathrm{~m}$.

对上述工况进行了一一计算, 限于篇幅我们只给出少部分数值模拟的波高平面分布图 (图 6). 通过对不 同情况数值计算结果的比较, 可以得到风浪场的如下特点: 无论是哪个风向, 在风力为 2 级时, 仅在湖区的 部分水域生成风浪. 例如, 在 ESE 向风的作用下, 仅在湖区的西北侧产生了风浪 (图 6); 湖区有效波高的最 大值为 $0.12-0.15 \mathrm{~m}$. 在风力为 4 级时, 在湖区的全部水域均生成了风浪 (图 6); 有效波高的最大值为 $0.27-0.29 \mathrm{~m}$. 在风力为 6 级时, 湖区有效波高的最大值为 $0.49-0.53 \mathrm{~m}$. 在风力为 7 级时, 湖区有效波高的 最大值为 $0.65-0.67 \mathrm{~m}$. 波高的最大值与风向的关系不密切.
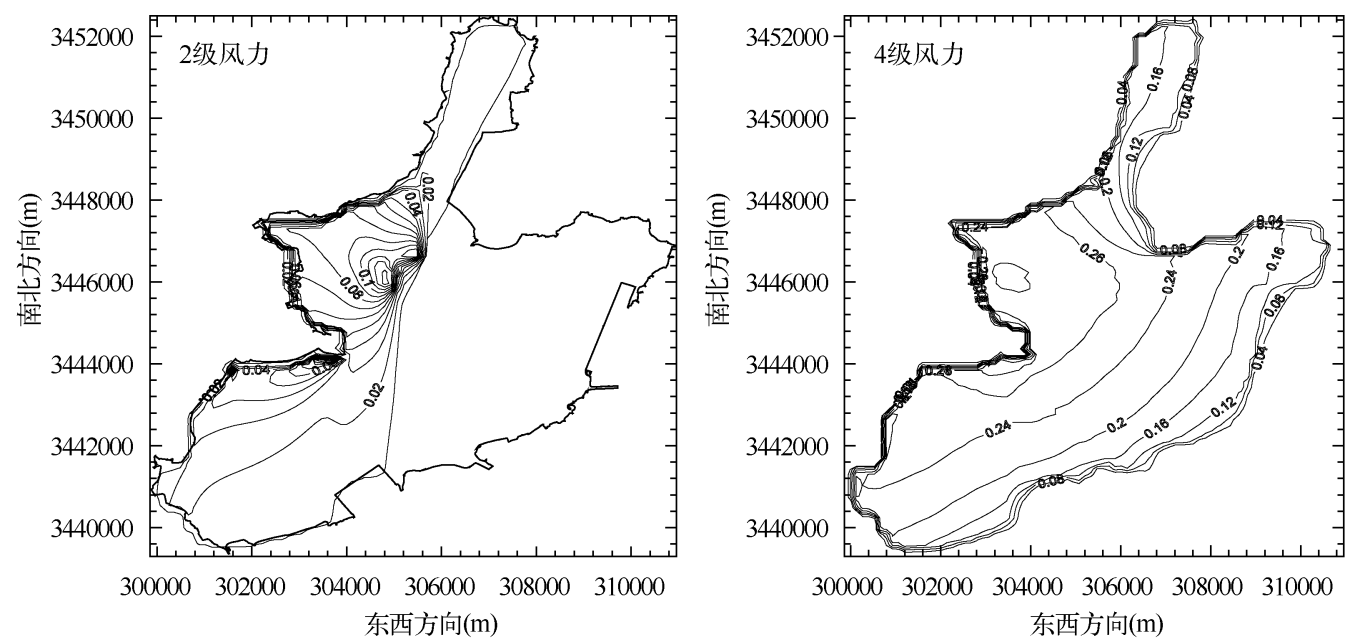

图 6 在遭遇不同风力的东南东向风( ESE) 时湖区的浪高平面分布图

Fig. 6 The distribution of wave height in the case of different grades of wind with the ESE direction

\section{2 不同重现期条件下风浪场的数值模拟}

当重现期为五十年一遇时, 淀山湖的平均水位为 $3.89 \mathrm{~m}$; 当重现期为一百年一遇时, 淀山湖的平均水位 为 $4.01 \mathrm{~m}$. 当重现期为五十年和一百年一遇时, 设计的最大风速分别为 $20.3 \mathrm{~m} / \mathrm{s}$ (相当于 8 级风力) 和 $21.4 \mathrm{~m} / \mathrm{s}$ (相当于 9 级风力 ${ }^{[19]}$. 就 $\mathrm{N} 、 \mathrm{NE} 、 \mathrm{E} 、 \mathrm{SE} 、 \mathrm{~S} 、 \mathrm{SW} 、 \mathrm{~W}$ 和 $\mathrm{NW}$ 八个不同的风向所产生的风浪场进行了数 值模拟. 在遭遇五十年一遇的风场和水位时, 湖区有效波高的最大值为 $1.24-1.36 \mathrm{~m}$; 在遭遇一百年一遇的 风场和水位时,湖区有效波高的最大值为 $1.27-1.39 \mathrm{~m}$.

\section{3 淀山湖风浪场特性的进一步讨论}

考虑到夏季的常风向为 ESE, 我们计算该风向的风场作用下, 夏季湖区波高的变化特点. 假定风速从 $1 \mathrm{~m} / \mathrm{s}$ 以整数间隔变化到 $10 \mathrm{~m} / \mathrm{s}$. 在风速为 1 和 $2 \mathrm{~m} / \mathrm{s}$ 时, 计算的有效波高的最大值分别为 $2.43 \times 10^{-5} \mathrm{~m}$ 和 $1.02 \times 10^{-4} \mathrm{~m}$, 非常微小. 因此,不统计风速为 1 和 $2 \mathrm{~m} / \mathrm{s}$ 时风浪场的计算结果. 结合 4.1 和 4.2 的计算结果, 列出不同风力时湖区波高的最大值 (表 1), 进而得到湖区波高的最大值随风速变化的关系曲线(图 7 ).

表 1 夏季在不同风力的风场作用下湖区波高最大值的统计表

Tab. 1 The statistical table of the maximum wave height in Lake Dianshan for different wind fields in summer

\begin{tabular}{lcccccccccccccc}
\hline 风速 $(\mathrm{m} / \mathrm{s})$ & 2.5 & 3.0 & 4.0 & 5.0 & 6.0 & 6.7 & 7.0 & 8.0 & 9.0 & 10.0 & 12.5 & 15.5 & 20.3 & 21.4 \\
\hline 有效波高 $(\mathrm{m})$ & 0.14 & 0.15 & 0.16 & 0.21 & 0.26 & 0.28 & 0.29 & 0.33 & 0.38 & 0.42 & 0.52 & 0.67 & 1.36 & 1.39 \\
\hline
\end{tabular}




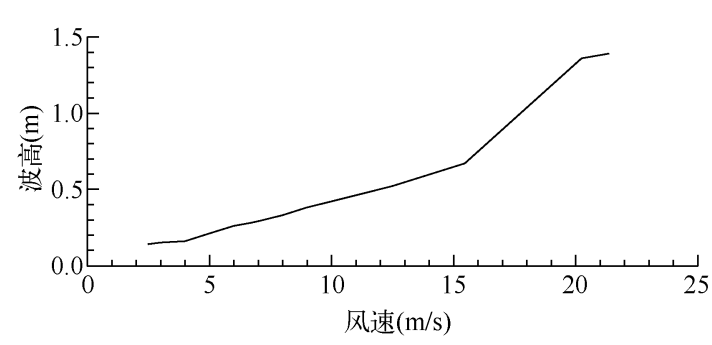

图 7 数值模拟的淀山湖有效波高的最大 值随风速的变化图

Fig. 7 The calculated maximum of significant wave height with the change of wind speed

\section{5 结语和讨论}

本文通过将基于 SWAN 模型计算的波高和现 场观测值的比较, 说明利用 SWAN 模型能较好地模 拟淀山湖的风浪场, 这表明 SWAN 模型的模拟结果 是可信的. 从而, 可以利用风浪数值模型— SWAN 模型这一较为简便、直接的手段探讨研究淀 山湖水域风浪场的变化特点. 较为系统地计算了不 同水位和不同风场的组合作用下淀山湖水域的风 浪场. 通过对计算结果的讨论, 说明利用 SWAN 模 型计算的湖区有效波高的最大值随风速的变化规 律是合理的. 计算结果可以为淀山湖防汛工程的规 划和设计以及环境保护提供参考. 因此,本文的方法可以推广应用到其它内陆湖泊风浪要素的计算.

下一步研究将继续探讨 SWAN 模型在内陆湖泊的适用性, 特别是当波要素如波高和波周期较大时, 通 过将数值计算结果和观测结果进行比较, 可以更好地验证模型的计算精度, 进而可以更为系统和有效地模 拟计算内陆湖泊的风浪场.

\section{6 参考文献}

[1] 乔树梁, 杜金曼, 陈国平等. 风浪特性及风浪要素的计算. 水利水运科学研究, 1996, 3: 189-198.

[2] 胡维平, 胡春华, 张发兵等. 太湖北部风浪波高计算模式观测分析. 湖泊科学, 2005, 17(1): 41-46.

[3] 张洪生, 于小伟, 周华伟. 太湖风浪预测系统应急完善技术报告. 上海: 上海交通大学, 2009 .

[ 4 ] 中华人民共和国水利部. 堤防工程设计规范. 北京: 中国水利水电出版社, 1999.

[ 5] 文圣常, 余宙文. 海浪理论与计算原理. 北京: 科学出版社, 1984.

[ 6 ] Hasselmann K. On the non-linear transfer in a gravity wave spectrum. Journal of Fluid Mechnics, Part 1, Genearl theory, 1962, 12: 481-500.

[ 7 ] Hasselmann K. On the non-linear transfer in a gravity wave spectrum. Journal of Fluid Mechnics, Part 2, Conservation theory, wave-particle correspondence, irreversibility, 1963a, 15: 273-281.

[ 8 ] Hasselmann K. On the non-linear transfer in a gravity wave spectrum. Journal of Fluid Mechnics, Part 3, Evaluation of energy flux and sea-swell interactions for a Neuman spectrum, 1963b, 15: 385-398.

[ 9 ] Hasselmann S, Hasselmann K, Allender JH et al. Computations and parameterizations of the linear energy transfer in a gravity wave spectrum, II , Parameterizations of the nonlinear transfer for application in wave models. Journal of Physical Oceanography, 1985, 15: 1378-1391.

[10] The SWAN team. SWAN Technical documentation. Delft University of Technology, 2007.

[11] 李一平, 逢 勇, 刘兴平等. 太湖波浪数值模拟. 湖泊科学, 2008, 20(1): 117-122.

[12] Booij N, Ris RC, Holthuijsen LH. A third-generation wave model for coastal regions 1: model description and validation. Journal of Geophysical Research, 1999, 104 ( C4 ) : 7649-7666.

[13] Ris RC, Holthuijsen LH, Booij N. A third-generation wave model for coastal regions 2: verification. Journal of Geophysical Research, 1999, 104(C4) : 7667-7681.

[14] Lin WQ, Sanford LP, Suttles SE. Wave measurement and modeling in Chesapeake Bay. Continental Shelf Research, 2002, 22 : 2673-2686.

[15] Ou SH, Liau JM, Hsu TW et al. Simulating typhoon waves by SWAN wave model in coastal waters of Taiwan. Ocean Engineering, 2002, 29: 947-971.

[16] 徐福敏, 张长宽, 茅丽华等. 一种浅水波浪数值模型的应用. 水动力学研究与进展, 2000( $\mathrm{A}$ 辑), 15(4) : 429-434.

[17] 中国科学院南京地理与湖泊研究所. 太湖. 北京: 海洋出版社, 1993.

[18] 徐祝龄. 气象学. 北京: 高等教育出版社, 1994: 32-33.

[19] 张洪生, 文武健. 淀山湖堤防规划设计波浪爬高的研究. 上海: 上海交通大学研究报告, 2009. 